\title{
The indirect boundary element method for the two-dimensional pressure- and gravity-driven free surface Stokes flow
}

\author{
M. A. Ponomareva, M. P. Filina \& V. A. Yakutenok \\ National Research Tomsk State University, Russia
}

\begin{abstract}
The indirect boundary element method (IBEM) is formulated for a two-dimensional Stokes flow with moving boundary in the presence of gravity. For simulation of the non-Newtonian fluid flow an iterative scheme for solving a nonlinear system of algebraic equation is used to calculate nonlinear viscous terms. The selected scheme requires values of pseudo forces in each boundary element and each internal collocation point obtained from boundary conditions (for velocities and tractions) and velocity field found at the previous iteration. Boundary element integrals are calculated analytically with a singularity extraction and internal cells integrals are evaluated by using the standard Gaussian quadratures. Internal cells sources are estimated numerically within a finite difference approach to velocity derivatives calculation. The Poiseuille flow of power law fluid in a channel is calculated for a benchmark of the IBEM algorithm. The accuracy and convergence tests are presented for a wide range of power law index (0.2-1.2). In the presence of a free surface, no analytical solution is available. The problem of channel filling with power law fluid is analyzed. The shape and the movement of the free surface are investigated through a special numerical front tracking algorithm based on mesh refinement for the free surface and for the solution domain. Elements of high performance computing are presented. The obtained results coincide with those of other authors. It confirms that the presented algorithm and corresponding software can be applied to study a number of low Reynolds flows, for example in a mould filling technology.

Keywords: indirect BEM, free surface, non-Newtonian fluid, Poiseuille flow, power law, channel filling, fountain flow, high performance computing, injection moulding.
\end{abstract}




\section{Introduction}

The fundamental principles of the boundary element method (the BEM) were formulated by Brebbia [1]. The BEM is constantly being developed and perfected; it is a powerful means of investigating different problems of continuum mechanics [2]. The BEM found a wide application in fluid dynamics [3-5]. In particular, a great number of viscous fluid flows with free surface have been investigated with low Reynolds numbers. Several practically important problems were discussed in papers [6-10]. Important results were successfully obtained by means of the indirect BEM for the flow of Newtonian fluid. Very often it is necessary to take into account the viscosity dependence on the shear rate, i.e. to consider the nonNewtonian fluid flow. This leads to the nonlinear equations in the partial derivatives in contrast to the linear Stokes equations for the Newtonian fluid. The realization of the boundary element method for nonlinear equations includes the calculation of the domain integral from the earlier unknown functions. As a result of discretization of the boundary integral equations one obtains a system of nonlinear algebraic equations. To solve it one should use the iteration methods. There exist different approaches to solve this problem $[11,12]$. One of the alternatives is the Dual Reciprocity Method (DRM) which is used together with the NewtonRaphson iterative technique. It turned out to be an efficient way for the numerical investigation of the deformation of a non-Newtonian drop in the Newtonian fluid flow [13]. The truncated power law model has been used. The authors succeeded in performing the calculations with the values of the power law index of $n=0.8$ and 0.6 .

In the present work a classical approach given in $[3,4]$ for the problems of the elasticity theory is used to calculating domain integral in order to take account of the non-Newtonian pseudo body forces. This approach is used together with the method of the simple iteration (Picard method). It differs by its simplicity and high reliability as compared with more complicated methods, but it requires longer expenditure of machine time. On the other hand it is known, that BEM has a high degree of parallelism [14]. This advantage was used to get the results of the present work. The convergence and accuracy of the developed method were tested while solving the problem of the non-Newtonian fluid flow in a planar channel (Poiseuille flow). The power law was used as a rheological model. In this case the problem has a simple analytical solution. On achieving an approximate solution by means of the BEM the boundary conditions on the outlet boundary were specified similarly as on the free surface (the components of tractions). The developed algorithm showed a high computational efficiency and the possibility of calculating the flows of the non-Newtonian fluid with a high degree of nonlinearity (the power law index $n=0.2$ ). A fountain flow of the power law fluid in a planar channel was studied as an application of the method to solve the practical problems $(n=0.5,0.8,1)$. The investigation of this flow is of importance to study the process of filling the moulds while processing the polymers [15]. The obtaining of stationary advancing front shapes and prediction of their dependence on the values of the governing parameters, including power law index, are also actual in our time $[16,17]$. 


\section{Governing equations and problem formulation}

The two-dimensional flow of the non-Newtonian fluid at low Reynolds numbers with the account of the gravity force is described by the Stokes equation system:

$$
\frac{\partial \sigma_{i j}}{\partial x_{j}}+\rho g_{i}=0, i, j=1,2,
$$

where $\sigma_{i j}=-p \delta_{i j}+\tau_{i j}$ are the stress tensor components, $p$ is the pressure, $\rho$ is the density, $g_{i}$ are the components of the acceleration vector for the gravity force $\vec{g}, x_{i}$ are the Cartesian coordinates, $\delta_{i j}$ is the Kroneker delta, $\tau_{i j}$ are the stress tensor deviator components. The eqns (1) are used together with the continuity equation

$$
\frac{\partial u_{i}}{\partial x_{i}}=0
$$

where $u_{i}$ are the velocity vector components.

The non-Newtonian fluid behavior is described by the power law:

$$
\tau_{i j}=2 \eta \dot{e}_{i j}
$$

where $\eta=k \dot{\gamma}^{n-1}$ is the effective viscosity coefficient, $k$ is the power law consistency, $n$ is the power law index, $\dot{\gamma}=\left(2 \dot{e}_{i j} \dot{e}_{j i}\right)^{\frac{1}{2}}$ is the deformation rate intensity, $\dot{e}_{i j}=\frac{1}{2}\left(\frac{\partial u_{i}}{\partial x_{j}}+\frac{\partial u_{j}}{\partial x_{i}}\right)$ are the components of the rate-of-strain tensor.

The components of the velocity vector $u_{i}$ or the components of the surface traction vector $t_{i}=\sigma_{i j} n_{j}$ can be specified on the boundary of the flow domain. Here $n_{j}$ are the components of the outward unit normal vector to the boundary.

In order to pass to the dimensionless variables the following scales are adopted: $L$ for the coordinates (a characteristic length of the domain occupied by a fluid), $U$ for the velocity (a characteristic velocity), $k(U / L)^{n}$ for the pressure.

Using the dimensionless variables the eqn (1) will take the form:

$$
\frac{\partial \sigma_{i j}}{\partial x_{j}}+\mathrm{St}_{i}=0
$$

where $\mathrm{St}_{i}=\operatorname{St}\left(\vec{g} \cdot \vec{e}_{i}\right) /|\vec{g}|, \mathrm{St}=(\rho|\vec{g}| L) \cdot(L / U)^{n}$ is the modified Stokes number, $\vec{e}_{i}$ are the unit vectors of the Cartesian coordinate system. Eqns (2) and (3) do not change their external view, but now $\eta=\dot{\gamma}^{n-1}$ is the dimensionless coefficient of the effective viscosity. As a result the flow is determined by the two parameters St and $n$ and also by the domain configuration. Of course, if there is no account of 
the other forces in the boundary conditions, for example, the surface tension forces. This fact occurs for high viscosity fluids [15].

Eqn (4) is transformed to the form:

$$
\frac{\partial \sigma_{i j}^{N}}{\partial x_{j}}=\frac{\partial}{\partial x_{j}}\left[2(1-\eta) \dot{e}_{i j}\right]
$$

where $\sigma_{i j}^{N}=-p_{m} \delta_{i j}+2 \dot{e}_{i j}$ is a linear part of the stress tensor, $p_{m}=p-\mathrm{St}_{k} x_{k}$ $(k=1,2)$ is the modified pressure. The pressure $p_{m}$ is introduced to eliminate the constant term $\mathrm{St}_{i}$ in eqn (4). For this purpose the potential of the gravity force $\mathrm{St}_{k} x_{k}$ is applied. Its value equals to $-\mathrm{St} \cdot x_{2}$ if the gravity is opposite to $\vec{e}_{2}$.

\subsection{The flow of the non-Newtonian fluid in a channel (the Poiseuille flow)}

The problem of the non-Newtonian fluid flow in a planar channel is considered as a test. The flow rate is considered to be given. The channel width is chosen as a characteristic length, the average flow velocity is a characteristic velocity.

Let a boundary value problem be formulated. It is necessary to solve eqn (5) for the domain and the boundary conditions shown in fig. 1 (left). The velocity vector components are specified on the inlet boundary in accordance with the power law rheology analytical solution:

$$
u_{1}=0, u_{2}\left(x_{1}\right)=\frac{2 n+1}{n+1}\left(1-\left|2 x_{1}\right|^{\frac{n+1}{2}}\right) .
$$

The no slip conditions are set on the solid walls:

$$
u_{i}=0 .
$$

The traction vector components are specified on the outlet boundary. While using the momentum conservation eqn (5) one should set the linear part of the traction vector:

$$
t_{i}^{N}=\sigma_{i j}^{N} n_{j},
$$

which is bound with the total traction vector by the relationship:

$$
t_{i}=\sigma_{i j} n_{j}=\left(\sigma_{i j}^{N}+\tau_{i j}^{N N}\right) n_{j},
$$

where $\tau_{i j}^{N N}=2(\eta-1) \dot{e}_{i j}$ is a nonlinear part of the stress tensor.

To consider the problem of the planar channel: $n_{1}=0, n_{2}=1$ and accounting for (6) it is easy to get:

$$
t_{1}^{N}=\sigma_{12}^{N}=2 \dot{e}_{12}=\frac{\partial u_{2}}{\partial x_{1}}=-\frac{2 n+1}{n}\left(2\left|x_{1}\right|\right)^{\frac{1}{n}} \frac{x_{1}}{\left|x_{1}\right|}, \quad t_{2}^{N}=\sigma_{22}^{N}=-p+2 \frac{\partial u_{2}}{\partial x_{2}}=0 .
$$

Here the lack of the gravity force and the additivity of the pressure value are taken into account which allows to specify $p=0$ on the outlet bound. 
As a result of solving the problem by using the IBEM a velocity profile $u_{2}$ close to eqn (6) is to be obtained. Also for the algorithm verification the total traction vector is calculated and compared with its analytical solution:

$$
t_{1}=\sigma_{12}=\sigma_{12}^{N}+\tau_{12}^{N N}=2 \eta \dot{e}_{12}=-\left|\frac{\partial u_{2}}{\partial x_{1}}\right|^{n} \frac{x_{1}}{\left|x_{1}\right|}=-2\left(\frac{4 n+2}{n}\right)^{n} \cdot x_{1}, \quad t_{2}=0 .
$$

Thus, the linear profile for $t_{1}$ is to be obtained for any $n$ value.

\subsection{Filling a channel with the non-Newtonian fluid (the fountain flow)}

The formulation of the problem on filling a vertical channel with the non-Newtonian fluid is given in fig. 1 (right). The rate flow of the fluid at the inlet into the channel is considered to be given. The difference from the previous case lies in the fact that the outlet boundary is substituted by a free surface. The boundary conditions at the inlet and on the solid walls are specified as usual by the relations (6) and (7). Thus, we also do not use symmetry conditions and calculate both sides. The components of the traction vector are specified on the free surface with account of the pressure modifications:

$$
t_{i}=\mathrm{St}_{k} x_{k} n_{i} .
$$

The outside pressure is considered to be equal to zero, as its value does not affect the solution of the problem.

The following expression is obtained for the linear part of the traction vector from eqn (12) with account of eqns (8) and (9):

$$
t_{i}^{N}=\mathrm{St}_{k} x_{k} n_{i}-\tau_{i j}^{N N} n_{j} .
$$

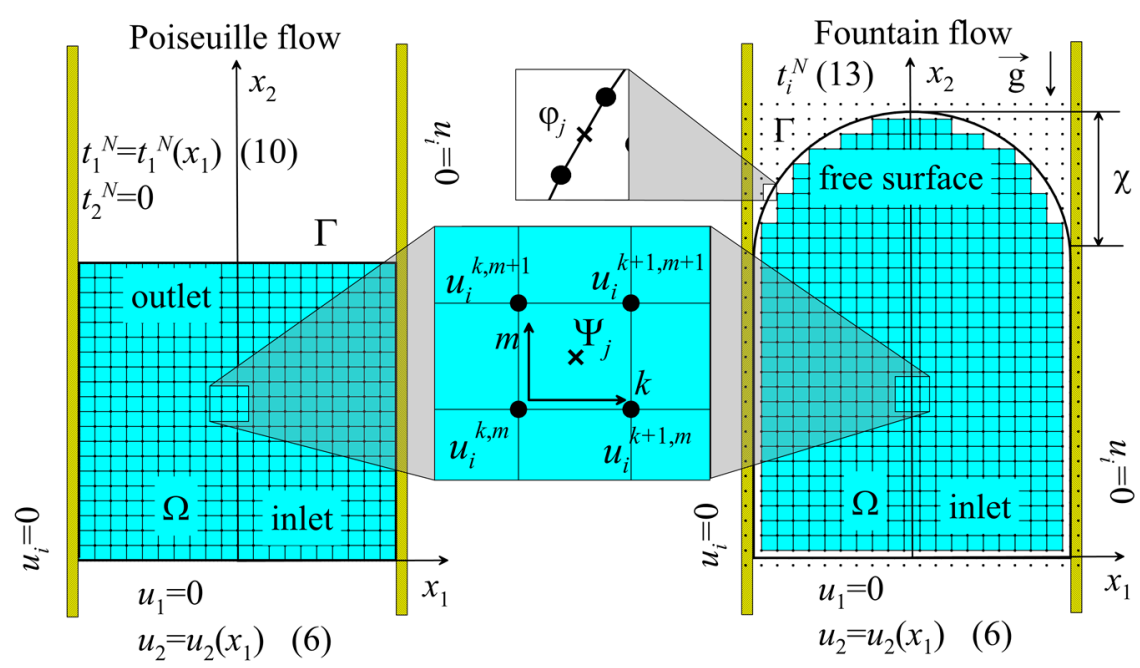

Figure 1: The solution domain and discretization. 
Eqn (13) is a dynamic boundary condition on the free surface if the equation of the momentum conservation is used in the form of eqn (5). In addition, the free boundary obeys the kinematic condition in the Lagrangian form:

$$
\frac{d x_{i}}{d t}=u_{i}
$$

Conditions of eqn (14) are used to find the shape of the free boundary. As an initial condition it is necessary to set the shape of the boundary at the initial moment of time.

\section{Boundary integral equations}

Eqn (5) is represented in the form:

$$
\frac{\partial \sigma_{i j}^{N}}{\partial x_{j}}=\Psi_{i}
$$

where $\Psi_{i}=-\partial \tau_{i j}^{N N} / \partial x_{j}$ is a nonlinear vector function which will be considered to be the density of the sources distributed about the flow domain. Then, according to the postulates of the IBEM [3,4] one can write down

$$
\begin{aligned}
& u_{i}(x)=\int_{\Gamma} G_{i j}(x, \xi) \varphi_{j}(\xi) d \Gamma(\xi)+\int_{\Omega} G_{i j}(x, z) \Psi_{j}(z) d \Omega(z), \\
& t_{i}^{N}(x)=\int_{\Gamma} F_{i j}(x, \xi) \varphi_{j}(\xi) d \Gamma(\xi)+\int_{\Omega} F_{i j}(x, z) \Psi_{j}(z) d \Omega(z),
\end{aligned}
$$

where $\varphi_{j}(\xi)$ is the density of the fictitious sources distributed along the boundary $\Gamma$ of $\Omega$ domain occupied by the fluid, fig. 1 . The functions $G_{i j}$ and $F_{i j}$ are the singular solutions of the Stokes equations obtained by Ladyzhenskaya [18] and they are determined by formulae:

$$
G_{i j}(x, \xi)=-\frac{1}{4 \pi}\left(\delta_{i j} \ln \frac{1}{r}+\frac{y_{i} y_{j}}{r^{2}}\right), \quad F_{i j}(x, \xi)=\frac{y_{i} y_{j} y_{k} n_{k}(x)}{\pi r^{4}},
$$

where $y_{i}=x_{i}-\xi_{i}, r=y_{i} y_{i}^{1 / 2}$.

The properties of the integral eqns (16) are described in detail in [3, 4] including the case of $x \in \Gamma$. If the values of $t_{i}^{N}(x), u_{i}(x)$ are specified on the boundary $\Gamma$, the eqns (16) make it possible to get the values of the unknown boundary forces $\varphi_{j}(\xi)(\xi \in \Gamma)$. But since $\Psi_{i}(z)(z \in \Omega)$ functions are not known before, one can use an iteration method for this purpose.

\section{Numerical implementation}

For a numerical solution of eqns (16) the constant elements and the constant internal cells are applied. The boundary of the flow domain $\Gamma$ is divided into $N$ elements. The function $\varphi_{j}(\xi)$ is considered to be constant on every element. The 
flow domain is covered by a grid of straight lines which are parallel to the coordinate axes $x_{1}, x_{2}$, fig. 1 . To compute the value of the domain integral only the grid cells belonging to the domain $\Omega$ are applied (the ray tracing algorithm realized to solve of a geometry problem). The $\Psi_{j}(z)$ functions are considered to be constant inside the cell. The discretized-form eqns (16) acquire the form:

$$
\begin{aligned}
& u_{i}\left(x^{p}\right)=\sum_{q=1}^{N} \varphi_{j}^{q} \Delta G_{i j}^{p q}+\sum_{k} \sum_{m} \Psi_{j}^{k m} \Delta G_{i j}^{p k m}, \\
& t_{i}^{N}\left(x^{p}\right)=\sum_{q=1}^{N} \varphi_{j}^{q} \Delta F_{i j}^{p q}+\sum_{k} \sum_{m} \Psi_{j}^{k m} \Delta F_{i j}^{p k m},
\end{aligned}
$$

where

$$
\Delta G_{i j}^{p q}=\int_{\Delta \Gamma^{q}} G_{i j}\left(x^{p}, \xi\right) d \Gamma(\xi), \quad \Delta F_{i j}^{p q}=\int_{\Delta \Gamma^{q}} F_{i j}\left(x^{p}, \xi\right) d \Gamma(\xi),
$$

$\Delta G_{i j}^{p k m}=\int_{\Delta \Omega^{k m}} G_{i j}\left(x^{p}, z\right) d \Omega(z), \Delta F_{i j}^{p k m}=\int_{\Delta \Omega^{k m}} F_{i j}\left(x^{p}, z\right) d \Omega(z), x^{p}$ is the middle of the element $p$, i.e. the node $p$.

To compute $2 N$ of the unknown values $\varphi_{j}^{q}$ one should take the $2 N$ of eqns (18) corresponding to the $N$ elements on which $u_{i}\left(x^{p}\right)$ or $t_{i}^{N}\left(x^{p}\right)$ are specified. The coefficients of the obtained system of the linear algebraic equations $\Delta G_{i j}^{p q}$ and $\Delta F_{i j}^{p q}$ for the case of the constant elements may be computed analytically. The computing technology is given by Banerjee and Butterfield [4] for the linear elasticity theory equations. The local Cartesian coordinate system $\eta_{i}=e_{i j}\left(x_{i}-x_{j}^{p}\right)$ is introduced for this purpose. The coordinate system origin is in the node $p$, and the direction of the axis $\eta_{1}$ coincides with that of the outward normal vector to the element $q$. Here $e_{i j}$ are the directive cosines of the axes $\eta_{i}$. As a result the following expressions are obtained:

$$
\begin{aligned}
& \Delta G_{i j}^{p q}=\frac{1}{4 \pi}\left\{\delta_{i j}(\ln r-1) \eta_{2}-\eta_{1}\left(e_{1 j} e_{2 i}+e_{2 j} e_{1 i}\right) \ln r-e_{2 j} e_{2 i}\left(\eta_{2}-2 \eta_{1} \Theta\right)\right\}_{q}^{q+1}, \\
& \Delta F_{i j}^{p q}=\frac{1}{2 \pi}\left\{e_{1 j} e_{1 i}\left[\vec{n}_{1}(\Theta+\sin \Theta \cos \Theta)+\vec{n}_{2} \sin ^{2} \Theta\right]+\left(e_{1 j} e_{2 i}+e_{2 j} e_{1 i}\right)\left[\vec{n}_{1} \sin ^{2} \Theta+\right.\right. \\
& \left.\left.+\vec{n}_{2}(\Theta-\sin \Theta \cos \Theta)\right]+e_{2 j} e_{2 i}\left[\vec{n}_{1}(\Theta-\sin \Theta \cos \Theta)-\vec{n}_{2}\left(\sin ^{2} \Theta-2 \ln r\right)\right]\right\}_{q}^{q+1},
\end{aligned}
$$

where $\vec{n}_{i}=e_{i j} n_{j}$ are the components of the outward normal to the element $p$ in the coordinate system $\eta_{i} ; r, \theta$ are polar coordinates, fig. 2 .

In the singular case $(p=q)$ we have the following:

$$
\Delta G_{i j}^{q q}=\frac{\Delta S_{q}}{4 \pi}\left\{\delta_{i j}\left[\ln \left(\frac{\Delta S_{q}}{2}\right)-1\right]-e_{2 j} e_{2 i}\right\}, \quad \Delta F_{i j}^{q q}=\frac{1}{2} \delta_{i j},
$$

where $\Delta S_{q}$ is the $q$ element length. 


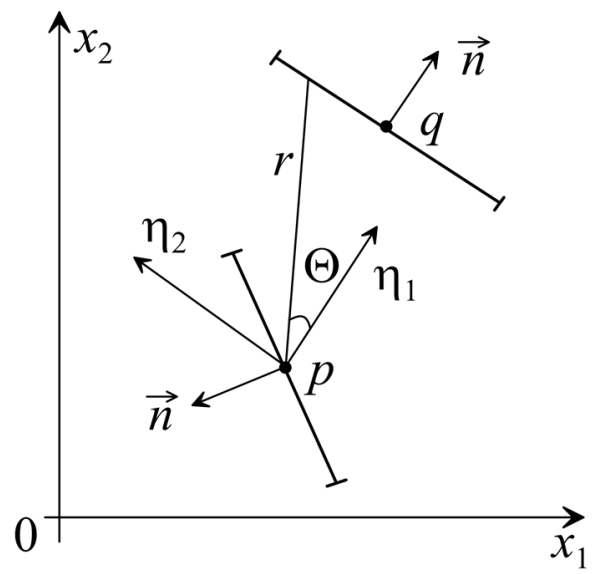

Figure 2: The local Cartesian coordinate system.

To compute the domain integrals $\Delta G_{i j}^{p k m}$ and $\Delta F_{i j}^{p k m}$ the standard quadrature Gaussian formulae are used without the singularities extraction. The singularities in these integrals have the forms $\ln r$ and $1 / r$. Consequently, while integrating over the domain they exist in the usual sense. Such approach considerably simplifies the solution algorithm. During the process of computing the quadrature formulae with 64 nodes are applied. To compute $\Psi_{j}^{k m}$ in the centres of the cells the velocity values $u_{i}^{k m}$ are preliminarily computed in the grid nodes $(k, m)$ (fig. 1), in accordance with the first eqn (18). At the first iteration $\Psi_{j}^{k m}$ values are calculated either from the Newtonian velocity field or taken from the previous time step with extrapolation to additional cells. The system of the linear algebraic eqns (18) is solved to determine $\varphi_{j}^{q}$ by the Gauss standard method. The $\Psi_{j}^{k m}$ values from the previous iteration are used further.

The computed $u_{i}^{k m}$ values make it possible to compute all the derivatives $\left(\partial u_{i} / \partial x_{j}\right)^{k m}$ in the grid nodes. For this purpose the central differences in the internal nodes and backward-forward differences in the boundary nodes are applied. Further the $\left(\tau_{i j}^{N N}\right)^{k m}$ values which are completely determined by the $\left(\partial u_{i} / \partial x_{j}\right)^{k m}$ values are computed. The $\Psi_{j}^{k m}$ functions themselves in the centres of the cells $(k, m)$ are computed by the finite-difference method with the use of the computed $\left(\tau_{i j}^{N N}\right)^{k m}$ in the grid nodes. Then, the system of eqns (18) is solved again to find the new $\varphi_{j}^{q}$ and so on. Consequently, the method of simple iteration has been realized.

The dynamic boundary condition has the form of eqn (13) on the free surface. The latter means that it is necessary to compute the non-linear part of the stress 
tensor $\tau_{i j}^{N N}$ in the nodes of the elements placed on the free surface. The $\tau_{i j}^{N N}$ values are completely determined by the $\dot{e}_{i j}$ values. But it is extremely difficult to compute $\dot{e}_{i j}$ on the curvilinear free surface by means of the finite-difference method. Therefore another method is used. Its main idea is shown for the elasticity theory problems in $[3,4]$. If the unit vectors of the Cartesian coordinate system $\vec{i}_{1}$, $\vec{i}_{2}$ are taken as the normal vectors to the boundary elements, we obtain:

$$
\begin{aligned}
& \vec{n}=\vec{i}_{1}: \quad t_{1}^{N}=\sigma_{1 j}^{N} n_{j}=\sigma_{11}^{N}=-p+2 \dot{e}_{11}, \\
& \vec{n}=\vec{i}_{2}: \quad t_{1}^{N}=\sigma_{1 j}^{N} n_{j}=\sigma_{12}^{N}=2 \dot{e}_{12}, \quad t_{2}^{N}=\sigma_{2 j}^{N} n_{j}=\sigma_{22}^{N}=-p+2 \dot{e}_{22} .
\end{aligned}
$$

The second eqn (18) for $t_{i}^{N}$ makes it possible to compute $\sigma_{11}^{N}, \sigma_{12}^{N}$ and $\sigma_{22}^{N}$ with the special normal selection shown above. Further, we can find:

$$
\dot{e}_{12}=\frac{\sigma_{12}^{N}}{2}, \quad \dot{e}_{11}=\frac{\sigma_{11}^{N}-\sigma_{22}^{N}}{4} .
$$

Using the continuity eqn (2) $\dot{e}_{22}=-\dot{e}_{11}$ and, naturally, $\dot{e}_{21}=\dot{e}_{12}$ are obtained. The same approach may be applied while computing $\tau_{i j}^{N N}$ in the nods of the grid inside the flow domain. The comparison with the finite-difference method based on the computation of velocities showed that the results practically coincide in accuracy but requires more CPU time.

Having found the velocity values in the edges of the elements belonging to the free surface, its new shape is computed. For this purpose a kinematic condition (14) is applied. A finite difference scheme for eqn (14) as follows:

$$
x_{i}^{n+1}=x_{i}^{n}+u_{i}^{n} \Delta t
$$

where $n$ is the number of the time step, $\Delta t$ is the time step, $i$ is the number of the element edge on the free surface. Hence, the problem with a moving free surface may be characterized as quasi-stationary, since the solution for the famous shape of the free boundary is used to find a new one.

\section{Results and discussion}

The system of the algebraic eqns (18) may be written in a matrix form as

$$
\mathrm{AX}=\mathrm{B}(\mathrm{X}) \text {, }
$$

where $\mathrm{A}$ is the matrix with the coefficients $\Delta G_{i j}^{p q}, \Delta F_{i j}^{p q}, \mathrm{X}$ is the vector of the unknown boundary sources $\varphi_{j}^{q}, \mathrm{~B}(\mathrm{X})$ is the vector of the nonlinear right part. The vector $\mathrm{B}(\mathrm{X})$ includes the nonlinear boundary conditions and the domain integrals from the internal sources $\Psi_{j}$. To solve this system the following variant of the simple iteration method is applied. Let at the $s^{\text {th }}$ and $(s-1)^{\text {th }}$ iterations 
there exist $\mathrm{X}$ and $\Psi_{j}$ values computed earlier. Also there is a computed value of the Euclidian norm of the residual vector:

$$
r=\|\mathrm{AX}-\mathrm{B}(\mathrm{X})\| .
$$

Further the following sequence of steps is realized:

1. the relaxation parameter $\omega$ is considered to be equal to 1 ;

2. the linearized system (20) is solved to obtain $X^{s+1}$ value;

3. the $\mathrm{X}^{s+1}$ is recalculated as $\mathrm{X}^{s+1}=\omega \mathrm{X}^{s+1}+(1-\omega) \mathrm{X}^{s}$;

4. internal sources are recalculated as $\Psi_{j}^{s+1}=\omega \Psi_{j}^{s}+(1-\omega) \Psi_{j}^{s-1}$;

5. the new values of $\Psi_{j}^{s+1}$ are computed in accordance with the old ones;

6. a new $r$ value is computed and compared with the old one;

7. if the new $r$ value is above the previous one the relaxation parameter decreases $(\omega=0.9 \omega)$ and we return to step 3 ( $\omega$ is limited by the value 0.1 ).

8. the condition to stop iterations is checked: $r<M \cdot 10^{-6}$, where $M$ is the total number of the internal cells.

This version of the simple iteration method turned out to be efficient in a wide range of power law index values. It was chosen on the basis of a great number of computational experiments. Decreasing $\omega$ occurs only in the case of low $n$.

\subsection{The Poiseuille flow}

The main criterion to validate the computational algorithm is to compare it with the analytical solutions (6) and (11). The statement of the boundary value problem described in section 2.1 is specially used in order to imitate the boundary conditions of the problem with a free surface. The latter makes it possible to take account of all the nonlinear terms correctly. The numerical values of $u_{i}$ and $t_{i}$ are computed in the edges of the elements disposed on the outlet boundary. The deviation in the $L_{2}$ norm of the numerical solution from the exact one is calculated as follows

$$
e=\sqrt{\sum_{i=1}^{N_{\text {oultet }}}\left(u_{2 i}^{\text {analitic }}-u_{2 i}\right)^{2} / N_{\text {outlet }}},
$$

where $u_{2 i}^{\text {anal }}$ is the solution (6), $u_{2 i}$ is the calculated value, $N_{\text {outlet }}$ is the number of edges on the outlet boundary. The dependence of the deviation on the total number of elements is shown in fig. 3 (left) for different values of power law index. Whence, one can see that a rather good agreement may be reached at $N$ values $\approx 100$ even for the most unfavourable case $n=0.2$. One can see from fig. 3 (right) that the number of iterations is in accordance with the power-law index and it is high in the case of low $n$. The results for $n=0.2$ at $N=100$ are given in fig. 4 . The process of $u_{2}$ evolution during iterations, beginning from the Newtonian profile, is shown in the fig. 4 (left). As a result the analytical profile is almost achieved. At $n>0.2$ the profiles do not differ visually from solution (6). The comparison 
of the computed total tractions components with formula (11) shows their complete coincidence, fig. 4 (right). The values of $t_{1}^{N}$, which are specified as the boundary conditions, are shown in the same figure.
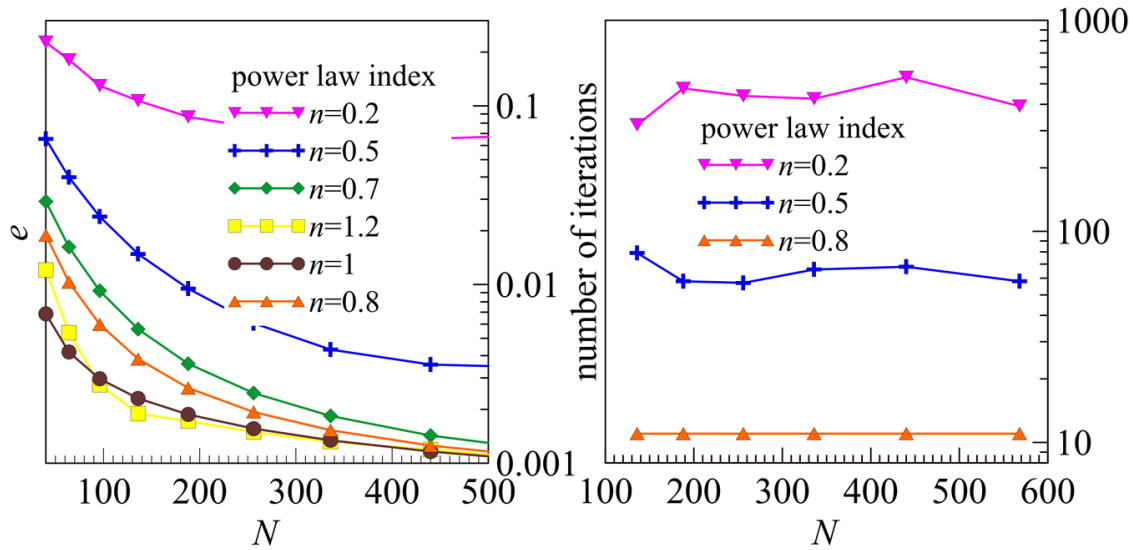

Figure 3: The accuracy and the number of iterations versus the boundary elements number.
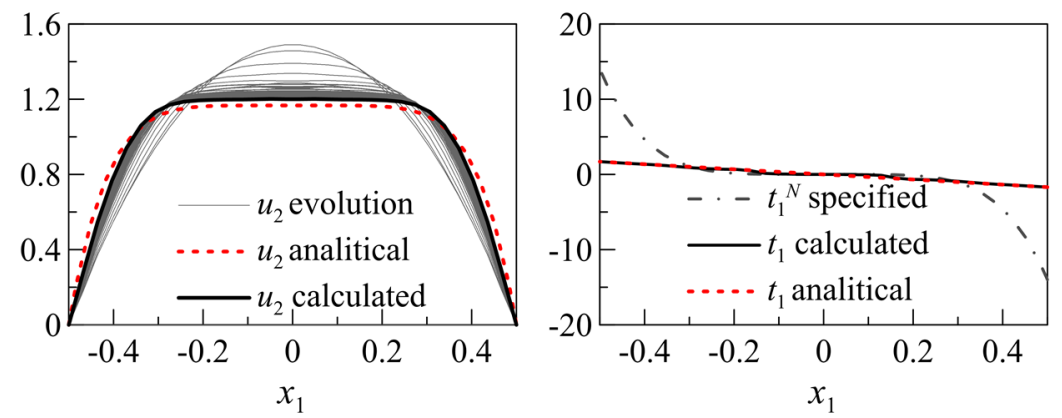

Figure 4: The velocity and the tractions profiles calculated for $n=0.2$ at $N=120$.

The analysis of the CPU time has been made while executing the different parts of the algorithm. For this purpose one node with two 6-core processors Intel ${ }^{\circledR}$ Xeon ${ }^{\text {TM }} 36702.99 \mathrm{GHz}$ of SKIF «Cyberia» cluster of National Research Tomsk State University was used for each calculation. The better way to solve the system of linear algebraic equations, for such fully populated matrix as in our case, is to use GETRF, GETRS routines from the LAPACK linear algebra package (Intel Math Kernel library). For example, this routines are up to five times more powerful as compared with standard codes SOLVE, DECOMP [19] even in one threading executing. The matrix assembly and the system of linear algebraic equations solving take almost all the computation time for the Newtonian case. For the non- 
Newtonian fluid these time expenses are negligible as compared with the computation time of the right part of eqn (20), namely of the internal sources estimation. The OpenMP technology was implemented to accelerate all the above algorithm parts. Figure 5 shows dependence of calculation time on the number of boundary elements during the sequential and parallel executing. The achieved speedup corresponds to the number of threads (12 for the above system). The static distribution of the loop iterations gives the highest performance while applying the OpenMP for the domain integral computation.

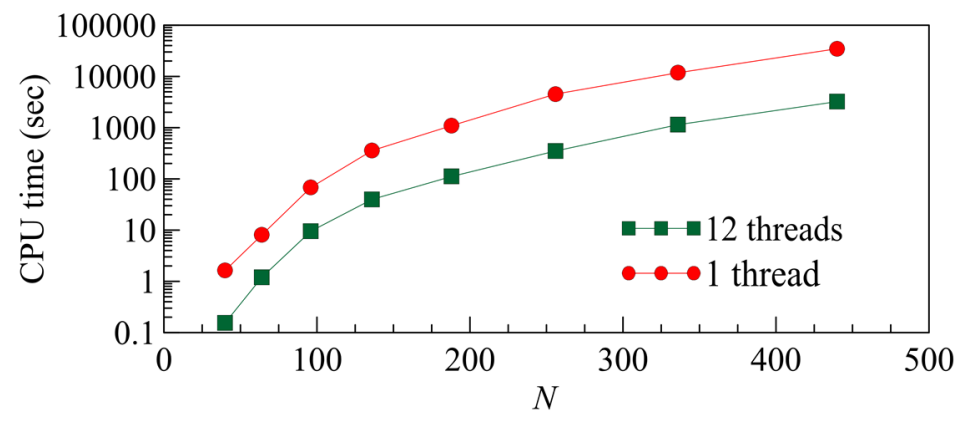

Figure 5: The CPU time versus $N$ at $n=0.5$ while using LAPACK and OpenMP during sequential and multithreading executing.

\subsection{The fountain flow}

At the initial time the fluid occupies a rectangular domain and the free surface is flat. The velocity profiles corresponding to this period are illustrated in fig. 6 . During the numerical simulation of the transient flow in a planar channel we deal with the moving front of the tracking line. The latter and the fountain flow behaviour lead to the necessity of the boundary element remeshing on the free surface. The algorithm is to increase continuously the number of elements under the continuous growth of the boundary length and its deformation. This algorithm together with the IBEM made it possible to investigate rather complex flows of the Newtonian fluid with moving free surface [6]. The IBEM application to the transient flow in a channel also gives great opportunities. Fig. 7 presents the dependence of such characteristic of fountain flow as the centreline front location $\chi$ (see fig. 1) on the Stokes number within the range $0 \leq \mathrm{St} \leq 1000$ for the Newtonian fluid. Here the comparison with the results of Mitsoulis [16] is presented. In [16] this problem was solved in steady state formulation by the FEM in the range $0 \leq \mathrm{St} \leq 160$. The results obtained for $n=0.5$ and $n=0.8$ are also illustrated in the same figure. As one can see from fig. 7 and fig. 8 for the nonNewtonian fluid the stationary advancing front in the absence of the gravity $(\mathrm{St}=0)$ has a more stretched shape as compared with the Newtonian one. At the same time the stretching increases while $n$ decreases as predicted by Mitsoulis [17]. Fig. 8 presents the stationary advancing front shapes. It is seen, if the gravity strongly affects the flow the non-linear features influence the $\chi$ value and the front 
shape to a small extent. The front shape is flatter as compared with the Newtonian one. There are no similar results for the simulation of the channel filling with the power law fluid in the presence of gravity.
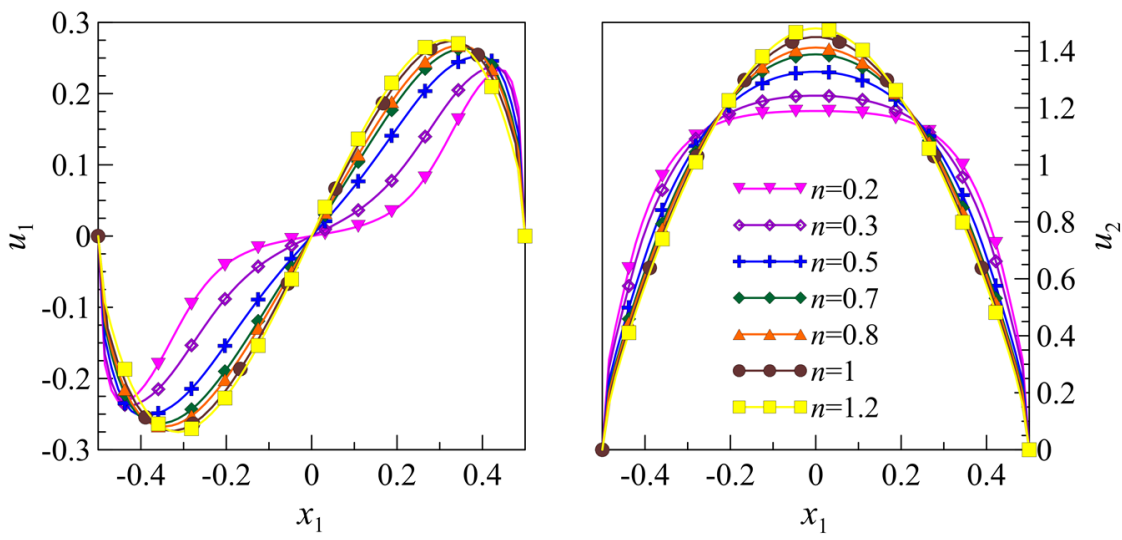

Figure 6: The unbended free surface velocity profiles at $t=0$.

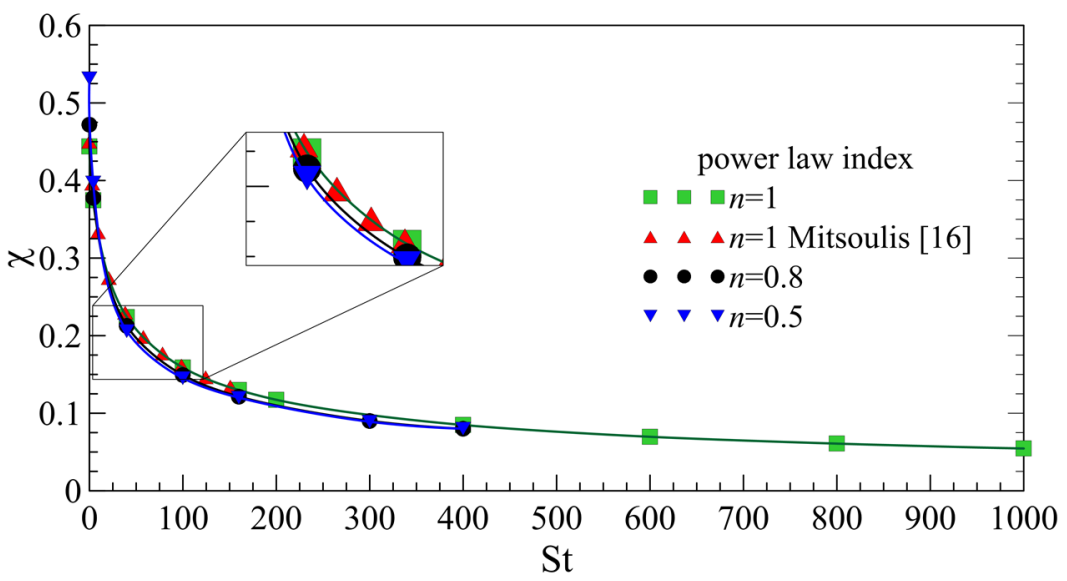

Figure 7: The dimensionless centreline front location $\chi$ versus the Stokes number.

Fig. 9 shows the dimensionless viscosity field inside the flow region at the fixed dimensionless time, when the free surfaces are stationary (all values of viscosity above 2 are red). One can see that the increase in the Stokes number influences not only the front shape, but also the viscosity distribution inside the flow domain. Note, that a sharp growth of the viscosity values is observed in the neighborhood of the centerline in accordance with the power law due to low shear rates in the vicinity of this line. Presented results are obtained for a total number of boundary elements about 250 and number of internal cells about 3000 in average. Mass loss obtained about $0.1 \%$. 


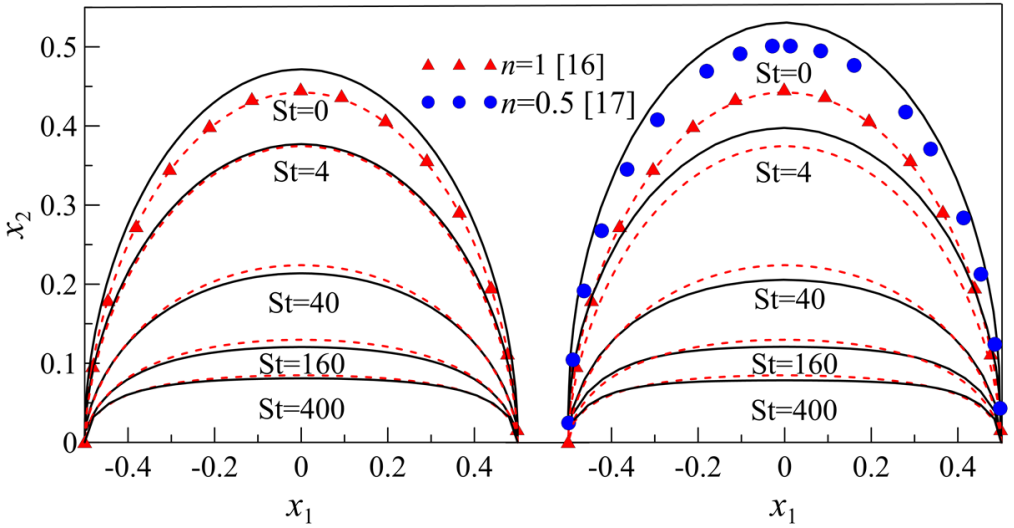

Figure 8: $\quad$ The advancing front shapes for $n=0.8$ (left) and $n=0.5$ (right): dashed red curves are the Newtonian ones; triangles (Mitsoulis [16]) for $n=1, \mathrm{St}=0$; circles (Mitsoulis [17]) for $n=0.5, \mathrm{St}=0$.
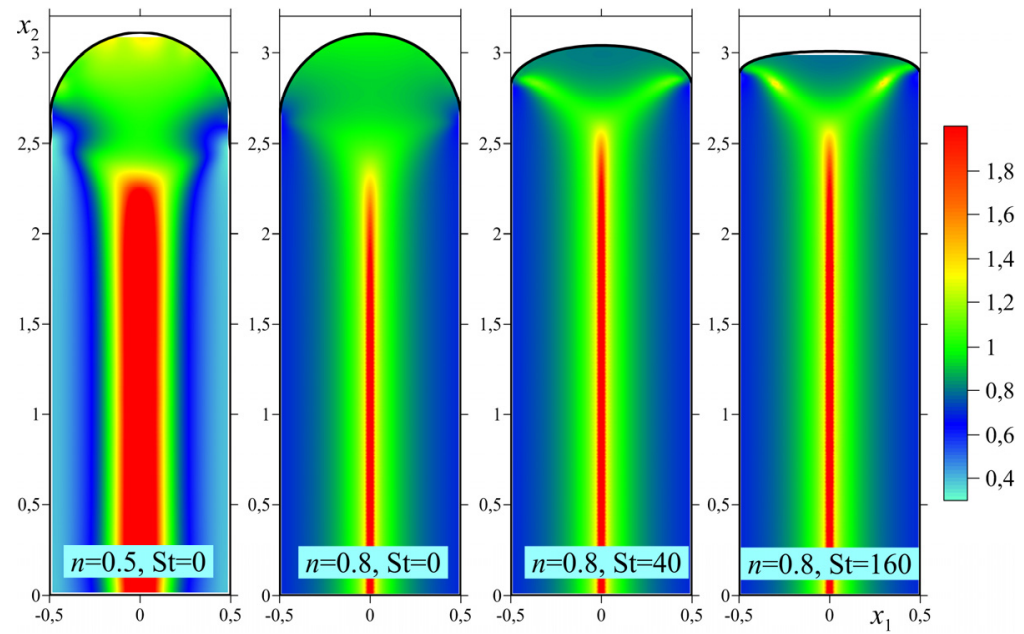

Figure 9: $\quad$ Free surface positions and viscosity field inside the flow domain at $t=2$.

\section{Conclusions}

The results of the present paper allow concluding that the IBEM is an efficient means to solve the problems dealing with the non-Newtonian fluids with a free surface. The constant elements and the internal cells provide an adequate accuracy of computations and decrease the expenditures on creation of the program code. As a result we have obtained a universal parallel program code for the numerical simulations of a class of problems associated with the two-dimensional creeping 
flows of the non-Newtonian fluid with free surfaces. The IBEM combined with the simple iteration method allows modelling the fluid flows at the power law index values down to 0.2 . For the guaranteed decrease in the residual it is necessary to apply an additional iteration process along relaxation parameter. The fountain flow of the shear-thinning fluid investigated in a wide range of the Stokes number changes for $n=0.5,0.8,1$. The non-linearity influencing on the flow under consideration described.

\section{Acknowledgements}

The work was supported by RF President's grant for young scientists No. MK3687.2014.1 and RFBR grants No. 14-08-31579 mol_a and No. 12-08-00313a. We also acknowledge the support of HPC SKIF-Cyberia (Tomsk State University).

\section{References}

[1] Brebbia C.A. The Boundary Element Method for Engineers, Pentech Press: London, 1978.

[2] Cheng A.H.-D. \& Chen D.T. Heritage and early history of the boundary element method. Engineering analysis with boundary elements, 6, pp. 268-302, 2005.

[3] Brebbia C.A., Telles J.C.F. \& Wrobel L.C. Boundary Element Techniques. Theory and applications in engineering, Sprinqer-Verlag: Berlin, 1984.

[4] Banerjee P.K. \& Butterfield R. Boundary Element Methods in Engineering Science, McGraf-Hill Book Company: UK, 1981.

[5] Pozrikidis C. Boundary Integral and Singularity Methods for Linearized Viscous Flow, Cambridge University Press: London, 1992.

[6] Ponomareva M.A., Yakutenok V.A. \& Shrager G.R. Stability of a Plane Jet of a Highly Viscous Fluid Impinging on a Horizontal Solid Wall. Fluid Dynamics, 46(1), pp. 44-50, 2011.

[7] Shrager G.R., Shtokolova M.N. \& Yakutenok V.A. Formation of the free surface of a viscous fluid volume inside a rotating horizontal cylinder. Fluid Dynamics, 44(2), pp. 322-327, 2009.

[8] Novoshintsev A.V., Shrager G.R. Yakutenok V.A. et al. Simulation of viscous liquid flow under the action of pressure drop with channel filling. Theoretical Foundations of Chemical Engineering, 43(3), pp. 325-334, 2009.

[9] Novoshintsev A.V., Shrager G.R. \& Yakutenok V.A. Numerical modeling of the outflow of a viscous liquid from a bulk mixer. Theoretical Foundations of Chemical Engineering, 40(6), pp. 626-632, 2006.

[10] Shrager G.R., Shtokolova M.N. \& Yakutenok V.A. Modeling of the viscous flow with a free surface inside a rotating horizontal cylinder. Theoretical Foundations of Chemical Engineering, 39(3), pp. 283-289, 2005. 
[11] Partridge P., Brebbia C.A. \& Wrobel L.C. The Dual Reciprocity Boundary Element Method, Southampton Computational Mechanics Publications, 1992.

[12] Nguen-Thien T., Trang-Cong T. \& Plan-Thien N. An improved boundary element method for analysis of profile polymer extrusion. Engineering Analysis with Boundary Elements, 20, pp. 81-89, 1997.

[13] Giraldo M., Power H., \& Flores W.F. Numerical simulation of the motion and deformation of a non-Newtonian shear-thinning drop suspended in a Newtonian circular Couette flow using DR-BEM. Engineering Analysis with Boundary Elements, 33, pp. 93-104, 2009.

[14] Gómez J.L. \& Power H. A parallel multipolar indirect boundary element method for the Neumann interior Stokes flow problem. International Journal of Numerical Methods in Engineering, 48, pp. 523-543, 2000.

[15] Tadmor Z. \& Gogos C.G. Principles of Polymer Processing, John Wiley \& Sons, Inc.: Hoboken, New Jersey, 2006.

[16] Mitsoulis E. Fountain Flow Revisited: The Effect of Various Fluid Mechanics Parameters. AIChE Journal, 56(5), pp. 1147-1162, 2010.

[17] Mitsoulis E. Fountain flow of pseudoplastic and viscoplastic fluid. Journal of Non-Newtonian Fluid Mechanics, 165, pp. 45-55, 2010.

[18] Ladyzhenskaya O.A. The Mathematical Theory of Viscous Incompressible Flow, Gordon \& Breach. 2nd ed.: New York, 1969.

[19] Forsythe G.E., Malcolm M.A. \& Moler C.B. Computer methods for mathematical computations, Prentice-Hall, Inc.: Englewood Cliffs, 1977. 